PHYSICAL REVIEW D 91, 129905(E) (2015)

\title{
Publisher's Note: Smooth bounce in the affine quantization of a Bianchi I model \\ [Phys. Rev. D 91, 124002 (2015)]
}

Hervé Bergeron, Andrea Dapor, Jean Pierre Gazeau, and Przemysław Małkiewicz

(Received 15 June 2015; published 26 June 2015)

This paper was published online on 1 June 2015 with incorrect author affiliations. The author list and corresponding affiliations should read as:

Hervé Bergeron, ${ }^{1, *}$ Andrea Dapor, ${ }^{2, \dagger}$ Jean Pierre Gazeau, ${ }^{3,4, \ddagger}$ and Przemysław Małkiewicz ${ }^{3,5,8}$

${ }^{1}$ ISMO, UMR 8214 CNRS, Univ Paris-Sud, 91405 Orsay, France

${ }^{2}$ University of Warsaw, Hoża 69, 00-681 Warsaw, Poland

${ }^{3}$ APC, UMR 7164 CNRS, Univ Paris Diderot, Sorbonne Paris Cité, 75205 Paris, France

${ }_{5}^{4}$ Centro Brasileiro de Pesquisas Fisicas, 22290-180 Rio de Janeiro, RJ, Brazil

${ }^{5}$ National Centre for Nuclear Research, Hoża 69, 00-681 Warsaw, Poland

The paper has been corrected as of 17 June 2015. The author affiliations are correct in the printed version of the journal. 